\title{
Evaluation of Treatment Response after Endoscopic Variceal Obturation with Abdominal Computed Tomography
}

Han Ah Lee, ${ }^{1}$ Hyun Gil Goh, ${ }^{1}$ Tae Hyung Kim, ${ }^{1}$ Young-Sun Lee, ${ }^{1}$ Sang Jun Suh, ${ }^{1}$ Young Kul Jung, ${ }^{1}$ Hyuk Soon Choi, ${ }^{1}$ Eun Sun Kim, ${ }^{1} \mathrm{Ji}$ Hoon Kim, ${ }^{1}$ Hyunggin An, ${ }^{2}$ Yeon Seok Seo, ${ }^{1}$ Hyung Joon Yim, ${ }^{1}$ Sung Bum Cho, ${ }^{3}$ Yoon Tae Jeen, ${ }^{1}$ Jong Eun Yeon, ${ }^{1}$ Hoon Jai Chun, ${ }^{1}$ Kwan Soo Byun, ${ }^{1}$ Soon Ho Um, ${ }^{1}$ and Chang Duck Kim ${ }^{1}$

Departments of ${ }^{1}$ Internal Medicine, ${ }^{2}$ Biostatistics, and ${ }^{3}$ Radiology, Korea University College of Medicine, Seoul, Korea

Backgrounds/Aims: Rebleeding of gastric varices (GVs) after endoscopic variceal obturation (EVO) can be fatal. This study was performed to evaluate the usefulness of computed tomography (CT) for the prediction of rebleeding after EVO GV bleeding. Methods: Patients who were treated with EVO for GV bleeding and underwent CT before and after EVO were included. CT images of the portal phase showing pretreatment GVs and feeding vessels, and nonenhanced images showing posttreatment cyanoacrylate impaction were reviewed. Results: Fifty-three patients were included. Their mean age was $60.6 \pm 11.6$ years, and 40 patients $(75.5 \%)$ were men. Alcoholic liver disease was the most frequent underlying liver disease (45.3\%). Complete impaction of cyanoacrylate in GVs and feeding vessels were achieved in 40 (75.5\%) and 24 (45.3\%) of patients, respectively. During the follow-up, GV rebleeding occurred in nine patients, and the cumulative incidences of GV rebleeding at 3, 6, and 12 months were $11.8 \%, 18.9 \%$, and $18.9 \%$, respectively. The GV rebleeding rate did not differ significantly according to the complete cyanoacrylate impaction in the GV, while it differed significantly according to complete cyanoacrylate impaction in the feeding vessels. The cumulative incidences of GV rebleeding at 3, 6, and 12 months were $22.3 \%, 35.2 \%$, and $35.2 \%$, respectively, in patients with incomplete impaction in feeding vessels, and there was no rebleeding during the follow-up period in patients with complete impaction in the feeding vessels $(p=0.002)$. Conclusions: Abdominal CT is useful in the evaluation of the treatment response after EVO for GV bleeding. Incomplete cyanoacrylate impaction in feeding vessels is a risk factor for GV rebleeding. (Gut Liver

\section{0;14:117-124)}

Key Words: Tomography; X-ray computed; Cyanoacrylates; Esophageal and gastric varices; Ethiodized oil

\section{INTRODUCTION}

Gastroesophageal varices (GEVs) are common complication of liver cirrhosis ${ }^{1}$ and bleeding from GEVs is a leading cause of mortality in patients with liver cirrhosis., ${ }^{2,3}$ Gastric varices (GVs) are present in $22 \%$ to $25 \%$ of patients with liver cirrhosis. ${ }^{4,5}$ Although bleeding from GVs occurs less frequently than from esophageal varices (EVs), bleeding from GVs shows a poorer prognosis with higher rebleeding and mortality rates. ${ }^{4,6,7}$

Current guidelines recommend endoscopic band ligation (EBL) as a treatment of choice for the management of bleeding and prevention of rebleeding from EVs. ${ }^{8,9}$ However, because of the differences in the depth of underlying submucosal layer over varices, size of varices, and surrounding environment, such as continuous exposure to gastric acid and peristalsis of GVs, EBL is insufficient for the management of bleeding from GVs. ${ }^{10}$ Also, several randomized controlled trials ${ }^{11-13}$ and meta-analyses ${ }^{14}$ suggested that endoscopic variceal obturation (EVO) showed significantly higher hemostasis rate and lower rebleeding rate than EBL. Therefore, current guidelines recommend EVO as a treatment choice for the management of bleeding from GVs. However, high incidence of rebleeding from GVs after EVO remains a problem. In previous studies, the rebleeding rate after EVO ranges from $7 \%$ to $41 \%$. $^{15-20}$

Although several previous studies suggested the predictive

\footnotetext{
Correspondence to: Yeon Seok Seo ${ }^{\mathrm{a}}$ and Sung Bum Cho ${ }^{\mathrm{b}}$

${ }^{a}$ Division of Gastroenterology and Hepatology, Department of Internal Medicine, Korea University College of Medicine, 145 Anam-ro, Seongbuk-gu, Seoul 02841, Korea

Tel: +82-2-920-6608, Fax: +82-2-953-1943, E-mail: drseo@korea.ac.kr

${ }^{\mathrm{b}}$ Department of Radiology, Korea University College of Medicine, 145 Anam-ro, Seongbuk-gu, Seoul 02841, Korea

Tel: +82-2-920-5555, Fax: +82-2-929-3796, E-mail: anglercho@gmail.com

Received on September 6, 2018. Revised on January 20, 2019. Accepted on January 21, 2019. Published online June $28,2019$. pISSN 1976-2283 eISSN 2005-1212 https://doi.org/10.5009/gnl18392

() This is an Open Access article distributed under the terms of the Creative Commons Attribution Non-Commercial License (http://creativecommons.org/licenses/by-nc/4.0) which permits unrestricted non-commercial use, distribution, and reproduction in any medium, provided the original work is properly cited.
} 
factors for rebleeding after EVO, such as liver function as expressed in the Child-Pugh class or Model for End-Stage Liver Disease score, accompanying hepatocellular carcinoma (HCC), and size of GVs, their results showed discrepancy. Some studies suggested that liver function is the significant predictor for rebleeding, ${ }^{17,21}$ while others did not. ${ }^{18,19,22}$ Similarly, GV size was the significant predictor for rebleeding in one study, ${ }^{16}$ while it was not associated with rebleeding in another study. ${ }^{22}$ In addition, although several studies suggested that accompanying HCC was significantly associated with rebleeding, ${ }^{12,16}$ the presence of malignancy was not related with rebleeding in another study. $^{18}$

Meanwhile, thanks to the recent improvement of computed tomography (CT), excellent visualization of GV as well as its feeding vessels has become available. Because various types of feeding vessels of GVs could be related to the different hemodynamics and treatment responses of $\mathrm{GVs},{ }^{23}$ detailed evaluation of GV and its feeding vessels by CT anatomy would give important information about prognosis after EVO. ${ }^{24}$ However, no studies have evaluated the degree of cyanoacrylate impaction in the GVs and feeding vessels.

We hypothesized that compact cyanoacrylate impaction in GV and its feeding vessels could be associated with recurrence and rebleeding of GV after EVO. Assessment of the effect of EVO on GVs and its feeding vessels not only by endoscopic findings, but also by CT images may improve prediction of recurrence and rebleeding of GVs after EVO. On the basis of this background, our study was performed to assess the usefulness of CT in evaluation of pretreatment hemodynamic features and treatment response after EVO to predict prognosis of GVs.

\section{MATERIALS AND METHODS}

\section{Patients}

Consecutive patients with GV bleeding who were treated with EVO between January 2006 and December 2016 and performed CT before and after EVO were included in this study. Patients who did not perform CT before and after EVO $(n=38)$, those with a previous history of endoscopic treatment for bleeding from GVs $(n=4)$ and those with accompanying portal vein invasion by HCC $(n=9)$ or other malignancy $(n=7)$ were excluded. In addition, patients with isolated GV type 2 (IGV2) were also excluded because the number was too small for analysis $(n=2)$. Liver cirrhosis was diagnosed based on histology and/or imaging studies. The study protocol conformed to the ethical guidelines of the Helsinki Declaration and was approved by the Institutional Review Board of Korea University Medical Center (IRB No. 2017AN0344). A waiver of consent was obtained and the patient records were anonymized and de-identified prior to analysis.

\section{Data collection}

Patient data regarding age, sex, and underlying liver disease (chronic hepatitis B, chronic hepatitis C, alcoholic liver disease, and others) were collected during hospitalization. The ChildPugh score was determined by applying Pugh's commonly used modification, which is based on the presence and severity of ascites and hepatic encephalopathy, prothrombin time prolongation, and serum bilirubin and albumin levels. ${ }^{25}$ Followup data on rebleeding and mortality were collected until 1 year after enrollment. Rebleeding was defined as any occurrence of hematemesis or a decrease in the hemoglobin level, with fresh melena after the successful control of the initial bleeding. ${ }^{9}$

\section{Classification of GVs}

GVs were classified according to the Sarin's classification ${ }^{4}$ as follows: GVs are divided into gastroesophageal varices (GOV) and IGV according to the association of GVs with EVs. GOV and IGV are divided into GOV1/GOV2 and IGV1/IGV2, respectively, according to their location within the stomach. GOV1 appears as continuations of EVs and extend for 2 to $5 \mathrm{~cm}$ below the gastroesophageal junction, along the lesser curvature of the stomach, while GOV2 extend below gastroesophageal junction into the fundus of the stomach. IGV1 is located in the fundus of the stomach and IGV2 is located anywhere in the stomach or intestine as isolated ectopic varices. GOV2 and IGV1 are also called as fundal varices.

\section{Endoscopic treatment}

In Korea University Medical Center, we perform esophagogastroduodenoscopy (EGD) in 6 hours if acute upper gastrointestinal bleeding is suspected. When the evidence of active or recent (e.g., stigmata) variceal bleeding is detected in the EGD, we ask for specialist endoscopic treatment or balloon-occluded retrograde transvenous obliteration in 1 hour, depending on the decision of the hepatologists. Vasoactive drugs, such as terlipressin or somatostatin, are used before diagnostic endoscopy.

EVO was performed using an Olympus Q-260, H-260, and HQ-290 video endoscope (Olympus Optical Co. Ltd., Tokyo, Japan) and a 21-gauge disposable injection needle (Injektionsnadel mit Metallspitze; MTW Endoskopie Manufaktur, Wesel, Germany). The injection needle was rinsed with distilled water before injections. The GVs were punctured and $1-2 \mathrm{~mL}$ of the mixture of n-butyl-2-cyanoacrylate (Histoacryl $\mathrm{L}^{\circledR}$; Braun Surgical SA, Rubi, Spain) and Lipiodol Ultra-Fluide (Guerbet, Bois Cedex, France) in the ratio of 1 to 1 was injected, immediately followed by a second injection of $1.0 \mathrm{~mL}$ of distilled water to flush the remaining mixture from the catheter. The needle was then withdrawn, followed by flushing of the needle with distilled water to keep it patent. During each session, 1 to $5 \mathrm{~mL}$ of mixture was used. After each procedure, the endoscopist checked for the effectiveness of the injections by gentle palpation of the 
treated varices with a blunt catheter. A hard feeling of the varices on palpation was considered a sign of effectively obliterated varices. Endoscopy was repeated 1 to 3 days after the initial injection to further confirm complete obliteration. If necessary, injections were repeated. Follow-up endoscopies were done 4 weeks later and then at 3-month intervals. EVO was repeated until obliteration of the GVs was achieved.

\section{Treatment evaluation with abdominal CT}

Feeding vessels were classified into three groups as follows: feeding via left or right gastric vein, left gastric vein (right sided feeding vessels, RFV); via short or posterior gastric vein, short gastric vein (left sided feeding vessels, LFV); and via both RFV and LFV, both gastric veins (both sided feeding vessels, BFV) (Fig. 1). Due to a small number of patients with BFV ( $n=7)$, they were classified as LFV.

CT response after EVO was evaluated by one radiologist (S.B.C.) who was unaware of the results of endoscopy and follow-up data. CT images of portal phase showing pretreatment GVs and feeding vessels, and nonenhanced images showing posttreatment cyanoacrylate impaction were reviewed. CT scans within 3 months before EVO were used as pretreatment images, and posttreatment CT scans were obtained within 3 days after the EVO.

Complete impaction was defined as the compaction of histoacryl within the entire lumen of varices or feeding vessels without normal vasculature, shown in portal phase of CT image. CT responses were determined according to the degree of cyanoacrylate impaction in GVs and feeding vessels and patients were classified into four groups as follows: CT-response group (CTrG) 1, complete cyanoacrylate impaction in both GV and

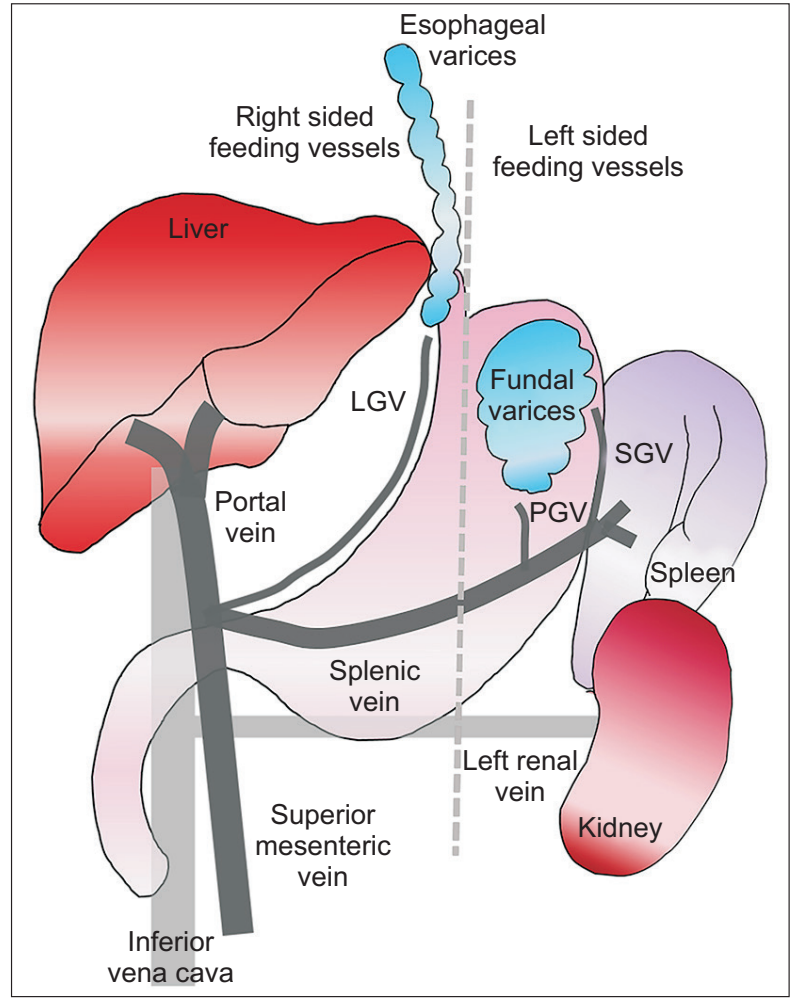

Fig. 1. Vascular anatomy of portal and systemic venous pathways potentially involved in gastric varices.

LGV, left gastric vein; PGV, posterior gastric vein; SGV, short gastric vein.
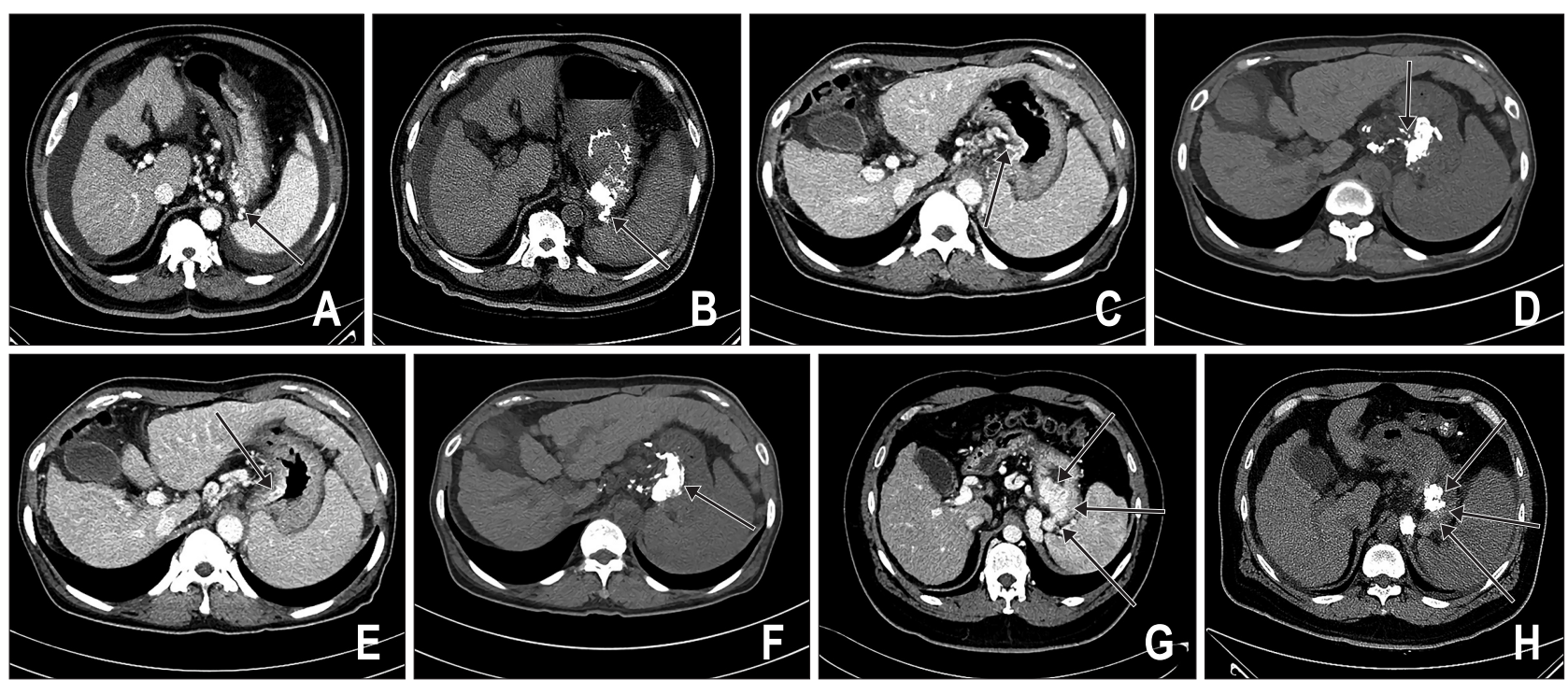

Fig. 2. Axial computed tomography (CT) images of various treatment responses after endoscopic variceal obturation (EVO) in patients with gastric variceal bleeding. Pretreatment portal phase dynamic CT image of liver (A, C). Posttreatment nonenhanced CT image shows complete (B) and incomplete cyanoacrylate impaction in feeding vessels (arrows) (D). Portal phase image shows gastric varices (GVs) (arrows) before EVO (E, G), and nonenhanced CT image shows GVs after EVO with complete (F) and incomplete cyanoacrylate impaction (H). 
feeding vessels; CTrG2, complete impaction in feeding vessels and incomplete impaction in GV; CTrG3, complete impaction in GV and incomplete impaction in feeding vessels; and CTrG4, incomplete impaction in both GV and feeding vessels. CT images revealing various treatment response of EVO in GVs and feeding vessels are shown in Fig. 2.

\section{Definition of rebleeding}

We used Baveno V definitions and criteria. ${ }^{26}$ Rebleeding was defined as clinically significant re-bleeding from portal hypertensive sources 5 days after EVO. Clinically significant rebleeding was defined as recurrent melena or hematemesis resulting in any of the following: hospital admission, blood transfusion, $3 \mathrm{~g}$ drop in hemoglobin, or death within 6 weeks.

\section{Statistics}

Statistical analyses were performed using SPSS software version 20.0 (IBM Corp., Armonk, NY, USA). Data were expressed as mean \pm standard deviation or number (\%). The Mann-Whitney $\mathrm{U}$ test and chi-square test were used to compare continuous and categorical variables, respectively. The cumulative rebleeding and mortality rates were determined by the Kaplan-Meier method, and the difference between groups was determined by the log-lank test. All tests were two-tailed, and p-values $<0.05$ were considered statistically significant.

\section{RESULTS}

\section{Baseline characteristics}

A total of 53 patients who were treated with EVO for GV

Table 1. Baseline Characteristics of All Included Patients Who Were Treated with Endoscopic Variceal Obturation for Gastric Variceal Bleeding

\begin{tabular}{lc}
\hline \multicolumn{1}{c}{ Variable } & All patients $(\mathrm{n}=53)$ \\
\hline Age, yr & $60.6 \pm 11.6$ \\
Male sex & $40(75.5)$ \\
Underlying liver disease & \\
Hepatitis B virus & $9(17.0)$ \\
Hepatitis C virus & $4(7.5)$ \\
Alcoholic liver disease & $24(45.3)$ \\
Other & $16(30.2)$ \\
Child- Pugh score & $7.6 \pm 1.9$ \\
Child- Pugh grade & \\
A & $14(26.4)$ \\
B & $31(58.5)$ \\
C & $8(15.1)$ \\
Hepatocellular carcinoma & $15(28.3)$ \\
Duration of follow-up, mo & $8.9 \pm 4.1$ \\
\hline
\end{tabular}

Data are presented as mean \pm SD or number $(\%)$. bleeding were included. Table 1 represents the baseline characteristics of all included patients. Mean age was $60.6 \pm 11.6$ years and 40 patients $(75.5 \%)$ were men. Alcoholic liver disease was the most frequent underlying liver disease (24 patients, 45.3\%). Child-Pugh score was $7.6 \pm 1.9$ and HCC were accompanied in 15 patients (28.3\%). Patients were followed for $8.9 \pm 4.1$ months after EVO (median, 12 months).

\section{Types of GVs and feeding and draining vessels}

The types of GV were GOV1, GOV2, and IGV1 in 17 (32.1\%), 17 (32.1\%), and 19 patients (35.8\%), respectively. The types of feeding vessel were RFV and LFV in 21 (39.6\%) and 32 patients (60.4\%), respectively. The types of feeding vessel differed significantly according to the type of GV as follows ( $<<0.001)$ : the feeding vessel was RFV in all patients with GOV1, RFV and LFV in four (23.5\%) and 13 patients (76.5\%), respectively, in GOV2, and it was LFV in all patients with IGV1.

\section{Treatment response in the abdominal CT after endoscop- ic treatment}

Complete impaction of cyanoacrylate in GVs and in the feeding vessels was achieved in 40 (75.5\%) and 24 of patients (45.3\%), respectively. The success rates of complete cyanoacrylate in GVs $(88.2 \%$, 58.8\%, and 78.9\% in patients with GOV1, GOV2, and IGV1, respectively, $\mathrm{p}=0.125$ ) and the feeding vessels (35.3\%, 41.2\%, and 57.9\% in patients with GOV1, GOV2, and IGV1, respectively, $\mathrm{p}=0.364$ ) were comparable among the three types of GVs.

Although the success rates of complete cyanoacrylate impaction in GVs was comparable in patients with GVs fed via RFV and those with GVs fed via LFV (85.7\% vs $68.8 \%, p=0.160$ ), the success rates of complete cyanoacrylate impaction in feeding vessels was significantly lower in patients with GVs fed via RFV than in those with GVs fed via LFV (28.6\% vs 56.2\%, $\mathrm{p}=0.048$ ).

Among the patients, 19 (35.8\%), 5 (9.4\%), 21 (39.6\%), and eight patients (15.1\%) were classified into CTrG1, CTrG2, CTrG3, and CTrG4, respectively. The proportions of patients in CT responses did not differ according to the types of GVs $(p=0.359)$ and the feeding vessels ( $p=0.089)$ (Fig. 3). Three patients had mild fever after EVO, and no other complications were observed.

\section{GV rebleeding}

During follow-up, GV rebleeding occurred in nine patients and the cumulative incidences of GV rebleeding at 1, 3, 6, and 12 months were $1.9 \%, 11.8 \%, 18.9 \%$, and $18.9 \%$, respectively. These were $0 \%, 5.9 \%, 12.6 \%$, and $12.6 \%$, respectively, in patients with GOV1, 0\%, 19.2\%, 26.0\%, and 26.0\%, respectively, in patients with GOV2, and 5.3\%, 10.5\%, 18.0\%, and 18.0\%, respectively, in patients with IGV1 $(p=0.632)$. The cumulative incidences of GV rebleeding at 1, 3, 6, and 12 months were $0 \%$, $4.8 \%, 15.3 \%$, and 15.3\%, respectively, in patients with GVs fed via RFV and 3.1\%, 16.9\%, 21.1\%, and 21.1\%, respectively, in 
A

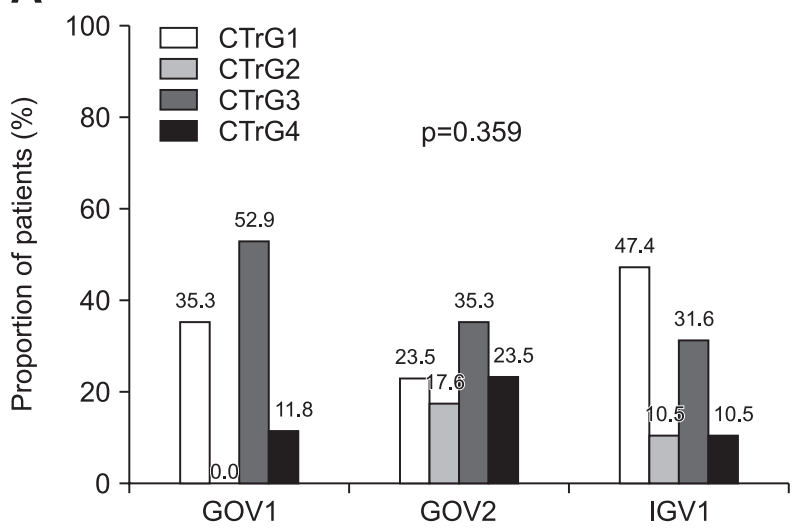

B

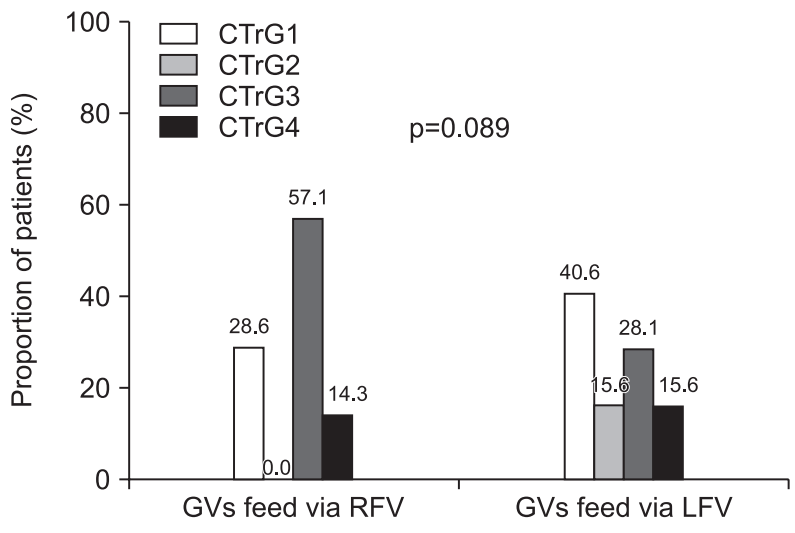

Fig. 3. Proportion of patients in the computed tomography (CT) response groups according to the type of gastric varices (A) and the type of feeding vessels (B).

CTrG, CT response group; CTrG1, complete cyanoacrylate impaction in both gastric varices (GV) and feeding vessels; CTrG2, complete impaction in feeding vessels and incomplete impaction in GV; CTrG3, complete impaction in GV and incomplete impaction in feeding vessels; CTrG4, incomplete impaction in both GV and feeding vessels; GOV1, gastroesophageal varices type 1; GOV2, gastroesophageal varices type 2; IGV1, isolated GV type 1; RFV, left or right gastric vein as a feeding vessel; LFV, short or posterior gastric vein as a feeding vessel.

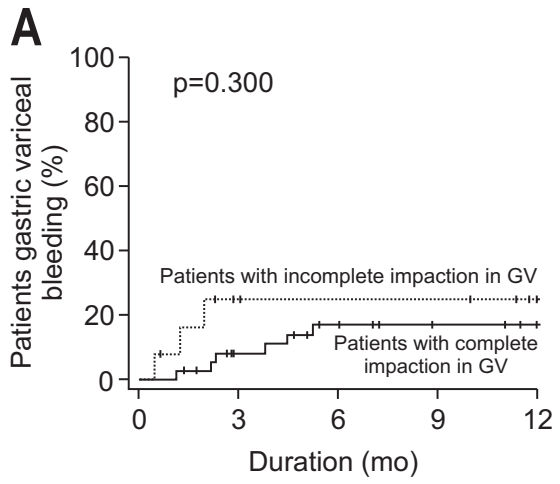

Patients at risk

Complete 40 Incomplete 13

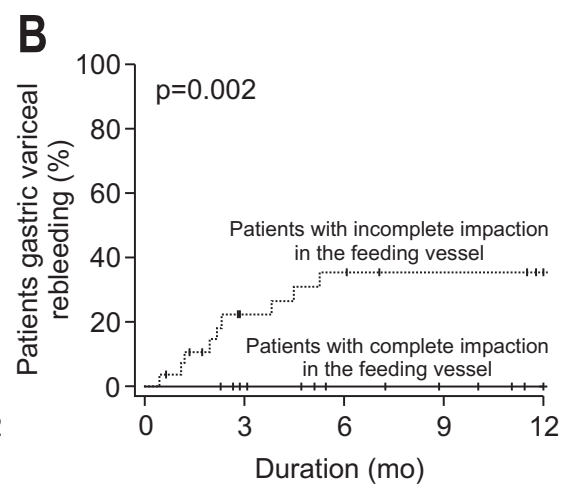

Patients at risk

20 Complete 24

3 Incomplete 29

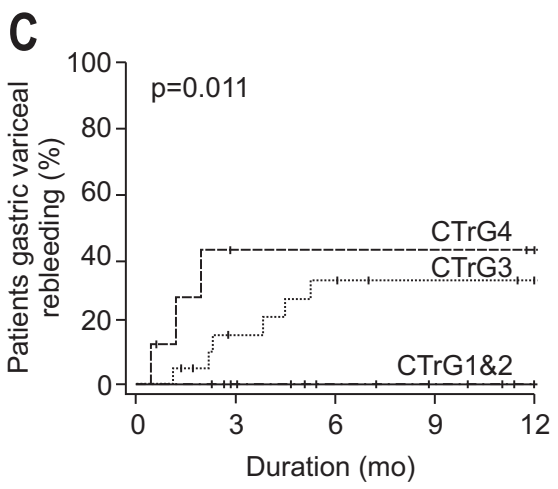

Patients at risk

$\begin{array}{rrrrrrr}20 & \text { CTrG1 } & 19 & 17 & 14 & 12 & 11 \\ 12 & \text { CTrG2 } & 5 & 4 & 3 & 3 & 1 \\ & \text { CTrG3 } & 21 & 15 & 12 & 10 & 9 \\ & \text { CTrG4 } & 8 & 3 & 3 & 3 & 2\end{array}$

Fig. 4. The cumulative gastric variceal rebleeding rates according to the complete cyanoacrylate impaction in gastric varices (A), the feeding vessels (B), and the computed tomography (CT) response group (C).

CTrG, CT response group; CTrG1, complete cyanoacrylate impaction in both gastric varices (GV) and feeding vessels; CTrG2, complete impaction in feeding vessels and incomplete impaction in GV; CTrG3, complete impaction in GV and incomplete impaction in feeding vessels; CTrG4, incomplete impaction in both GV and feeding vessels.

patients with GVs fed via LFV ( $\mathrm{p}=0.500$ ). GV rebleeding rates did not differ significantly according to the Child-Pugh class: the cumulative incidences of GV rebleeding at 1, 3, 6, and 12 months were $0 \%, 0 \%, 15.4 \%$, and $15.4 \%$, respectively, in patients with Child-Pugh class A, 0\%, 10.5\%, 14.8\%, and 14.8\%, respectively, in those with Child-Pugh class B, and 12.5\%, $37.5 \%$, 37.5\%, and 37.5\%, respectively, in those with ChildPugh class $C(p=0.106)$.

GV rebleeding rates did not differ significantly according to the complete cyanoacrylate impaction in GV: the cumulative incidences of GV rebleeding at 1, 3, 6, and 12 months were 7.7\%,
24.5\%, 24.5\%, and 24.5\%, respectively, in patients with incomplete cyanoacrylate impaction in GV and 0\%, 7.8\%, 16.6\%, and $16.6 \%$, respectively, in patients with complete cyanoacrylate impaction in GV ( $\mathrm{p}=0.300$ ) (Fig. $4 \mathrm{~A})$.

GV rebleeding rate differed significantly according to the complete cyanoacrylate impaction in feeding vessels: the cumulative incidences of GV rebleeding at 1, 3, 6, and 12 months were 3.4\%, 22.3\%, 35.2\%, and 35.2\%, respectively, in patients with incomplete cyanoacrylate impaction in feeding vessels and there was no rebleeding during the follow-up period in patients with complete cyanoacrylate impaction in feeding vessels 
( $\mathrm{p}=0.002)$ (Fig. 4B).

The cumulative incidences of GV rebleeding at 1, 3, 6, and 12 months were $0 \%, 15.3 \%, 32.3 \%$, and 32.3\%, respectively, in CTrG3 and 12.5\%, 41.7\%, 41.7\%, and 41.7\%, respectively, in CTrG4; there was no rebleeding during the follow-up period in patients with complete cyanoacrylate impaction in feeding vessels $(p=0.011$ ) (Fig. 4C). These patterns still remained in the subgroup analyses according to the Child-Pugh grade $(p=0.001)$ (Supplementary Fig. 1) and the type of GVs (Supplementary Fig. 2).

\section{Mortality}

During follow-up, six patients died with cumulative mortality rates of $1.9 \%, 7.9 \%, 10.1 \%$, and $13.1 \%$ at $1,3,6$, and 12 months, respectively. Mortality rate did not differ according to the types of GVs, feeding vessels and CTrG (Supplementary Fig. 3), while it differed significantly according to the Child-Pugh class: the cumulative mortality rates at $1,3,6$, and 12 months were $0 \%, 0 \%, 0 \%$, and $11.1 \%$, respectively, in patients with Child-Pugh class A, 0\%, 7.2\%, 7.2\%, and 7.2\%, respectively, in those with Child-Pugh class B, and 12.5\%, 27.1\%, 41.7\%, and $41.7 \%$, respectively, in those with Child-Pugh class C ( $p=0.020)$.

\section{DISCUSSION}

There have been various methods to evaluate the presence of GVs and the response of variceal treatment such as endoscopy, angiographic portography, and CT. Angiographic portography provides detailed hemodynamics of GVs, but it is an invasive procedure. ${ }^{26}$ In addition, because endoscopic findings are restricted to the mucosal layer, accurate evaluation of the state of submucosal vessels is not possible. CT scan permits comprehensive evaluation of varices and relevant vasculatures without invasive approaches. ${ }^{27-30}$ The greatest strength of this study is that, to the best of our knowledge, this is the first study that evaluated the treatment response of EVO with CT imaging. In favor of our hypothesis, the assessment of the effect of EVO by CT was helpful in the prediction of rebleeding after EVO.

In this study, we terminated EVO sessions when no remnant varices were left in endoscopic palpation. However, complete impaction of cyanoacrylate in the GVs and feeding vessels were achieved in only $45.3 \%$ and $75.5 \%$ of patients, respectively. As our results show, cyanoacrylate injection might not be sufficient in treating and feeding vessels though endoscopic palpation suggests complete impaction of GVs.

In our study, the only risk factor associated with the rebleeding was the presence of complete cyanoacrylate impaction in the feeding vessels. GVs develop as a portosystemic venous shunt based on anastomosis between inflowing and outflowing vessels. ${ }^{31}$ It has been generally believed that the complete obliteration of the GVs can prevent rebleeding, and it can be assessed by palpation of varices after cyanoacrylate injection. ${ }^{32}$
However, as GVs are formed from its inflowing routes, complete obliteration of feeding vessels by impaction of cyanoacrylate are needed to reduce rebleeding rate after EVO. Supporting this theory, this study shows that GV rebleeding rate differs significantly according to the presence of complete cyanoacrylate impaction in feeding vessels. Moreover, there was no rebleeding during the follow-up period in patients with complete cyanoacrylate impaction in feeding vessels.

To analyze the characteristics of GVs, we classified GV into two subgroups according to its major feeding vessel, RFV and LFV. One study suggested the classification of GVs into three subtypes in relation to the collateral veins: RFV predominant, LFV predominant, and the equal type, in which the RFV and LFV contributed about equally to the formation of varices. ${ }^{26}$ In that study, the types of feeding vessel were RFV, LFV, and equal type in $36.4 \%, 21.2 \%$, and $42.2 \%$ patients, respectively, and this result is quite similar to that of our study. In our study, the success rate of complete cyanoacrylate impaction in feeding vessels was significantly lower in patients with GVs fed via RFV than in those with GVs fed via LFV (28.6\% vs 56.2\%, $\mathrm{p}=0.048$ ), and it might because the diameter of RFV is larger than LFV. LFV commences from smaller tributaries than RFV. ${ }^{33}$

The type of GV was not associated with the rebleeding rate after EVO in this study and this result corresponds with several previous studies. ${ }^{17,19,21}$ However, we only enrolled small number of patients who performed CT for various purpose. Other studies also have limitations including small number of patients, evaluation of varices only by endoscopic findings, and lack of specific GV types (IGV1 or GOV1). ${ }^{19,34,35}$ Therefore, prospective, larger scale studies are needed for further verification. Also, presence HCC was not an independent risk factor for rebleeding in our study. A number of studies suggested that accompanying HCC was significantly associated with rebleeding, ${ }^{12,16,22}$ while the presence of malignancy was not related with rebleeding in another study. ${ }^{18}$ Meanwhile, a previous study suggested that presence of main portal vein thrombosis was one of the independent factors for rebleeding. ${ }^{21}$ Therefore, we excluded patients with HCC accompanied by portal vein thrombosis, and correlation between HCC and rebleeding was not apparent in this study. Likewise, presence of main portal vein thrombosis rather than HCC would affect rebleeding of GVs.

Our results showed a rebleeding rate of 18.9\% within 1 year. With the improvement of treatment technique and use of vasoactive drugs, rebleeding rate of GVs has decreased. ${ }^{22}$ However, it is somewhat higher than that of our previous study. ${ }^{36}$ It may be caused by selection bias, because when the treatment was considered to be successful, clinicians tended not to perform CT scans after EVO.

There are several limitations in this study. First, given its retrospective design, this study is subject to selection bias. Second, the number of enrolled patients was small. Another potential limitation was disparity between operator's skill. Because the 
skill of operator affect the outcome of EVO, these result are hard to be generalized in all clinical settings. Finally, CT itself has potential risks, such as the radiation hazards or contrast-induced nephropathy, and these risks should be considered.

In conclusion, abdominal CT is useful in the evaluation of treatment response after EVO. Because incomplete cyanoacrylate impaction in feeding vessels is a risk factor of GV rebleeding, detailed evaluation of feeding vessels on CT after EVO and determination of additional treatment is needed.

\section{CONFLICTS OF INTEREST}

No potential conflict of interest relevant to this article was reported.

\section{AUTHOR CONTRIBUTIONS}

Study concept and design: Y.S.S., S.B.C. Data acquisition: H.A.L., H.G.G., T.H.K., Y.S.L., S.J.S., Y.K.J., H.S.C., E.S.K., J.H.K., H.J.Y., Y.T.J., J.E.Y. Data analysis and interpretation: H.A.L., Y.S.S., H.A. Study supervision: H.J.C., K.S.B., S.H.U., C.D.K. Approval of final manuscript: all authors.

\section{ORCID} Hyun Gil Goh Tae Hyung Kim Young-Sun Lee Sang Jun Suh Young Kul Jung Hyuk Soon Choi Eun Sun Kim Ji Hoon Kim Hyunggin An Yeon Seok Seo Hyung Joon Yim Sung Bum Cho Yoon Tae Jeen Jong Eun Yeon Hoon Jai Chun Kwan Soo Byun Soon Ho Um Chang Duck Kim
Han Ah Lee https://orcid.org/0000-0003-4082-1121 https://orcid.org/0000-0002-5280-4794 https://orcid.org/0000-0002-7747-4293 https://orcid.org/0000-0001-6396-0859 https://orcid.org/0000-0003-2228-2793 https://orcid.org/0000-0002-6566-1382 https://orcid.org/0000-0002-4343-6950 https://orcid.org/0000-0002-5235-4332 https://orcid.org/0000-0001-6507-0614 https://orcid.org/0000-0002-0566-758X https://orcid.org/0000-0003-4171-6331 https://orcid.org/0000-0002-6036-2754 https://orcid.org/0000-0003-0259-0609 https://orcid.org/0000-0003-0220-3816 https://orcid.org/0000-0002-0510-7371 https://orcid.org/0000-0002-5539-361X https://orcid.org/0000-0003-4031-842X https://orcid.org/0000-0002-1369-0974 https://orcid.org/0000-0002-2829-6814

\section{REFERENCES}

1. D’Amico G, Pasta L, Morabito A, et al. Competing risks and prognostic stages of cirrhosis: a 25-year inception cohort study of 494 patients. Aliment Pharmacol Ther 2014;39:1180-1193.

2. Graham DY, Smith JL. The course of patients after variceal hemorrhage. Gastroenterology 1981;80:800-809.
3. Ginés P, Quintero E, Arroyo V, et al. Compensated cirrhosis: natural history and prognostic factors. Hepatology 1987;7:122-128.

4. Sarin SK, Lahoti D, Saxena SP, Murthy NS, Makwana UK. Prevalence, classification and natural history of gastric varices: a longterm follow-up study in 568 portal hypertension patients. Hepatology 1992;16:1343-1349.

5. Kim T, Shijo H, Kokawa H, et al. Risk factors for hemorrhage from gastric fundal varices. Hepatology 1997;25:307-312.

6. de Franchis R, Primignani M. Natural history of portal hypertension in patients with cirrhosis. Clin Liver Dis 2001;5:645-663.

7. Trudeau W, Prindiville T. Endoscopic injection sclerosis in bleeding gastric varices. Gastrointest Endosc 1986;32:264-268.

8. Garcia-Tsao G, Abraldes JG, Berzigotti A, Bosch J. Portal hypertensive bleeding in cirrhosis: risk stratification, diagnosis, and management. 2016 Practice guidance by the American Association for the study of liver diseases. Hepatology 2017;65:310-335.

9. de Franchis R; Baveno VI Faculty. Expanding consensus in portal hypertension: report of the Baveno VI Consensus Workshop. Stratifying risk and individualizing care for portal hypertension. J Hepatol 2015;63:743-752.

10. Seo YS. Prevention and management of gastroesophageal varices. Clin Mol Hepatol 2018;24:20-42.

11. Lo GH, Lai KH, Cheng JS, Chen MH, Chiang HT. A prospective, randomized trial of butyl cyanoacrylate injection versus band ligation in the management of bleeding gastric varices. Hepatology 2001;33:1060-1064.

12. Tan PC, Hou MC, Lin HC, et al. A randomized trial of endoscopic treatment of acute gastric variceal hemorrhage: N-butyl-2-cyanoacrylate injection versus band ligation. Hepatology 2006;43:690697.

13. Tantau M, Crisan D, Popa D, Vesa S, Tantau A. Band ligation vs. N-Butyl-2-cyanoacrylate injection in acute gastric variceal bleeding: a prospective follow-up study. Ann Hepatol 2014;13:75-83.

14. Qiao W, Ren Y, Bai Y, Liu S, Zhang Q, Zhi F. Cyanoacrylate injection versus band ligation in the endoscopic management of acute gastric variceal bleeding: meta-analysis of randomized, controlled studies based on the PRISMA statement. Medicine (Baltimore) 2015;94:e1725.

15. Caldwell SH, Hespenheide EE, Greenwald BD, Northup PG, Patrie JT. Enbucrilate for gastric varices: extended experience in 92 patients. Aliment Pharmacol Ther 2007;26:49-59.

16. Hou MC, Lin HC, Lee HS, Liao WC, Lee FY, Lee SD. A randomized trial of endoscopic cyanoacrylate injection for acute gastric variceal bleeding: $0.5 \mathrm{~mL}$ versus $1.0 \mathrm{~mL}$. Gastrointest Endosc 2009;70:668-675.

17. Marques P, Maluf-Filho F, Kumar A, Matuguma SE, Sakai P, Ishioka S. Long-term outcomes of acute gastric variceal bleeding in 48 patients following treatment with cyanoacrylate. Dig Dis Sci 2008;53:544-550.

18. Akahoshi T, Hashizume M, Shimabukuro R, et al. Long-term results of endoscopic Histoacryl injection sclerotherapy for gastric variceal bleeding: a 10-year experience. Surgery 2002;131(1 
Suppl):S176-S181.

19. Seewald S, Ang TL, Imazu H, et al. A standardized injection technique and regimen ensures success and safety of N-butyl-2-cyanoacrylate injection for the treatment of gastric fundal varices (with videos). Gastrointest Endosc 2008;68:447-454.

20. Rajoriya N, Forrest EH, Gray J, et al. Long-term follow-up of endoscopic Histoacryl glue injection for the management of gastric variceal bleeding. QJM 2011;104:41-47.

21. Hung HH, Chang CJ, Hou MC, et al. Efficacy of non-selective $\beta$-blockers as adjunct to endoscopic prophylactic treatment for gastric variceal bleeding: a randomized controlled trial. J Hepatol 2012;56:1025-1032.

22. Kang EJ, Jeong SW, Jang JY, et al. Long-term result of endoscopic Histoacryl (N-butyl-2-cyanoacrylate) injection for treatment of gastric varices. World J Gastroenterol 2011;17:1494-1500.

23. Kiyosue H, Ibukuro K, Maruno M, Tanoue S, Hongo N, Mori H. Multidetector CT anatomy of drainage routes of gastric varices: a pictorial review. Radiographics 2013;33:87-100.

24. Ito K, Matsutani S, Maruyama H, et al. Study of hemodynamic changes in portal systemic shunts and their relation to variceal relapse after endoscopic variceal ligation combined with ethanol sclerotherapy. J Gastroenterol 2006;41:119-126.

25. Pugh RN, Murray-Lyon IM, Dawson JL, Pietroni MC, Williams R. Transection of the oesophagus for bleeding oesophageal varices. Br J Surg 1973;60:646-649.

26. de Franchis R; Baveno V Faculty. Revising consensus in portal hypertension: report of the Baveno $\mathrm{V}$ consensus workshop on methodology of diagnosis and therapy in portal hypertension. J Hepatol 2010;53:762-768.

27. Watanabe K, Kimura K, Matsutani S, Ohto M, Okuda K. Portal hemodynamics in patients with gastric varices. A study in 230 patients with esophageal and/or gastric varices using portal vein catheterization. Gastroenterology 1988;95:434-440.

28. Chen TW, Yang ZG, Li X, Wang QL, Qian LL, Wang RR. Evaluation of entire gastric fundic and esophageal varices secondary to posthepatitic cirrhosis: portal venography using 64-row MDCT. Abdom Imaging 2010;35:1-7.

29. Nakayama Y, Imuta M, Funama Y, et al. CT portography by multidetector helical CT: comparison of three rendering models. Radiat Med 2002;20:273-279.

30. Ishikawa T, Ushiki T, Mizuno K, et al. CT-maximum intensity projection is a clinically useful modality for the detection of gastric varices. World J Gastroenterol 2005;11:7515-7519.

31. Mifune H, Akaki S, Ida K, Sei T, Kanazawa S, Okada H. Evaluation of esophageal varices by multidetector-row CT: correlation with endoscopic 'red color sign'. Acta Med Okayama 2007;61:247-254.

32. Johns TN, Evans BB. Collateral pathways in portal hypertension. Ann Surg 1962;155:838-845.

33. Cheng LF, Wang ZQ, Li CZ, Lin W, Yeo AE, Jin B. Low incidence of complications from endoscopic gastric variceal obturation with butyl cyanoacrylate. Clin Gastroenterol Hepatol 2010;8:760-766.

34. Liu YF, Zheng XD, Tajiri T, et al. The clinical significance of posterior gastric vein in portal hypertension as visualized in portograms. Nihon Ika Daigaku Zasshi 1988;55:209-212.

35. Kahloon A, Chalasani N, DeWitt J, et al. Endoscopic therapy with 2-octyl-cyanoacrylate for the treatment of gastric varices. Dig Dis Sci 2014;59:2178-2183.

36. Lee HA, Chang JM, Goh HG, et al. Prognosis of patients with gastric variceal bleeding after endoscopic variceal obturation according to the type of varices. Eur J Gastroenterol Hepatol 2019;31:211-217. 